mid-90s the gonorrhoea transmission was raised posing a serious public health problem especially among men who have sex with men (MSM). We examined if separate sexual networks exist of HIVinfected and of HIV-negative MSM in relation to transmission of gonorrhoea. Using molecular typing ofNeisseria gonorrhoeae (NG) we aimed to identify clusters of patients with specific NG strains and examined possible linkage to HIV infection and other characteristics.

Methods From July 2008 to August 2009, MSM visiting the Amsterdam outpatient clinic were recruited for a network study concerning sexually transmitted infections (STI). Inclusion criteria were age $\geq 18$ years, homosexual contact in the preceding 6 months, and giving informed consent. After screening for STI the participants answered questions regarding sexual behaviour, meeting places, and characteristics of sexual partners (up to 4 sex partners). Only patients with an anal or genital NG infection $(\mathrm{n}=246)$ were included in the current analysis. NG cultures were amplified and genotyped using a published NG-MLVA typing method.

Results Included patients (median age 36 years, IOR 30-42) were predominantly Dutch (83\%). Coinfections with chlamydia (28\%) and HIV (48\%) were common. Hierarchical cluster analysis of 246 MLVA profiles classified 152/246 MSM in 13 large clusters (5 to 36 patients) indicating the circulation and ongoing transmission of different NG strains in this population. In three clusters a significantly $(p<0.001)$ higher proportion of NG isolates had decreased susceptibility to cefotaxime. HIV infected MSM were older than HIV negative MSM and were evenly distributed over the NG clusters (see Abstract P1-S2.45 figure 1). There were no significant differences in age, nationality, nor in other coinfections between the various NG clusters.

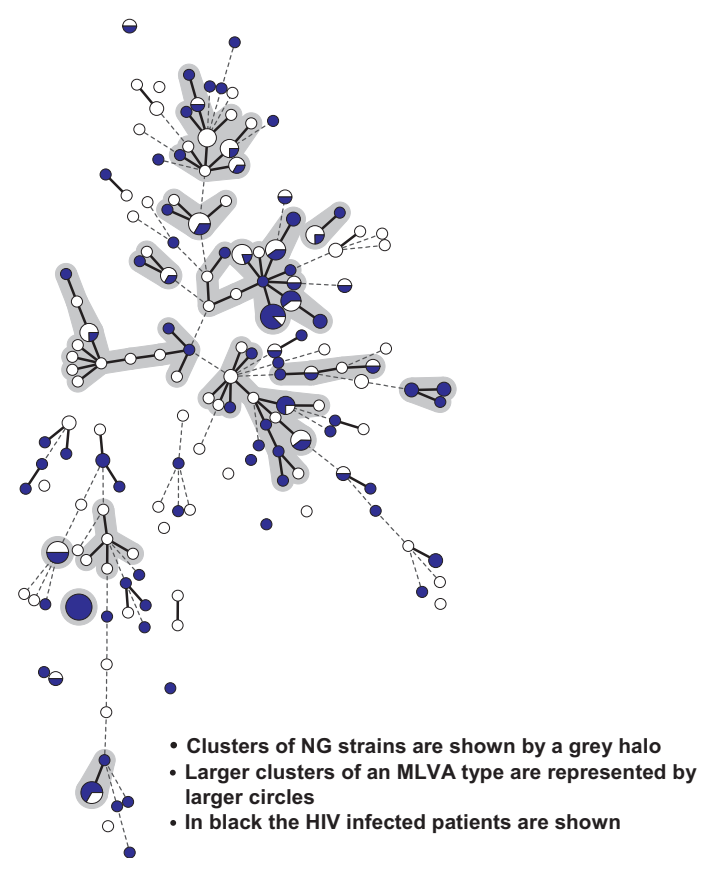

Abstract P1-S2.45 Figure 1 GGD.

Conclusions NG-MLVA revealed clusters of MSM reflecting distinct NG transmission networks in the Amsterdam population. As no NG clusters were identified that consisted predominantly of HIV infected or HIV negative MSM, it appears that there are no separate HIV infected and HIV negative sexual networks in the Amsterdam MSM population connected to gonorrhoea transmission.

\section{P1-S2.46 IS GROUP SEX A SETTING FOR INCREASED RISK FOR HIV AND OTHER STI AMONG HIV-NEGATIVE MEN WHO HAVE SEX WITH MEN?}

doi:10.1136/sextrans-2011-050108.103

J Heuker, I Stolte, U Davidovich. Public Health Service, Amsterdam, Netherlands

Background Transmission of HIV and other Sexually Transmitted Infections (STI) is ongoing in Western populations of Men who have Sex with Men (MSM). The main indicator of sexual risk is unprotected anal intercourse (UAI) with casual sex partners. It was suggested that group sex might be a high risk setting for HIV and STI transmission. Aim of the present study is to identify differences in sexual risk behaviour and STI-rates among MSM engaging in group sex compared to one-on-one casual sex.

Methods We used cross-sectional data derived through 6-monthly questionnaires and STI screening (infectious syphilis, gonorrhoea or Chlamydia) from the Amsterdam Cohort Studies (ACS) between December 2008 and December 2009. The study population $(n=310)$ consisted of HIV-negative MSM who reported having engaged in both group sex and one-on-one sex $(n=119)$ and MSM reporting one-on-one sex only $(n=191)$. To identify differences in sexual risk behaviour and STI-rates between MSM engaging in group sex and one-on-one sex, we used $\chi^{2}$ tests, Mann-Whitney $U$ tests and logistic regression analyses, corrected for repeated measures.

Results MSM engaging in both group sex and one-on-one sex had less anal intercourse (AI) during group sex (79/119; 66.4\%) compared to during one-on-one sex (106/119; 89.1\%). Moreover, they were less likely to have UAI during group sex compared to oneon-one sex (OR adj 0.44; 95\% CI 0.26 to 0.74 ). Men engaging in oneon-one sex only reported less AI (129/191; 67.5\%), while UAI-levels were similar (OR adj1.47; 95\% CI 0.82 to 2.62 ) compared to one-onone sex of men who also engage in group sex. Drug use during sex was associated with UAI (OR adj1.78; 95\% CI 1.03 to 3.09), independent of whether sex took place in a group sex or one-on-one sex setting. Finally, men engaging in group sex were more likely to be diagnosed with any STI (13.4\% vs $5.1 \%$; $\mathrm{p}=0.013)$ compared to men engaging in one-on-one sex only. For men engaging in one-on-one sex only, but not for men engaging in group sex, UAI was associated with being diagnosed with any STI (OR 8.44; $95 \%$ CI 2.0 to 35.2). Conclusions The group sex setting might not pose a threat for HIVinfection in MSM, as UAI-rates were lower during group sex compared to one-on-one sex. However, although they did not report more UAI, men engaging in group sex had higher STI-rates than men engaging in one-on-one sex only. This indicates that men engaging in group sex practice other sexual techniques than AI that contribute to acquiring STI.

\section{P1-S2.47 SEROSORTING AND SEXUAL RISK BEHAVIOUR DURING CASUAL SEX AMONG MSM - FROM ONE-NIGHT STANDS TO SEX BUDDIES}

doi:10.1136/sextrans-2011-050108.104

${ }^{1}$ I Stolte, ${ }^{1} \mathrm{~W}$ Van den Boom, ${ }^{2,3,4} \mathrm{~T}$ Sandfort, ${ }^{5} \mathrm{U}$ Davidovich. ${ }^{1}$ Public Health Service, Amsterdam, Netherlands; ${ }^{2}$ HIV Center for Clinical and Behavioural Studies, New York, USA; ${ }^{3}$ New York State Psychiatric Institute, New York, USA; ${ }^{4}$ Department of Psychiatry, Columbia University, New York, USA; ${ }^{3}$ Publich Health Service, Amsterdam, Netherlands

Background Among HIV-negative men who have sex with men (MSM), any incident of unprotected anal intercourse (UAI) between casual partners is usually regarded as risky for HIV. However, men are increasingly using knowledge of their casual partner's HIVstatus to reduce the risk for HIV during UAI (serosorting). Since familiarity between casual partners may lead to higher levels of UAI 
and serosorting, we examined how often men have UAI and report serosorting with three casual partner types that differ in the degree of familiarity.

Methods We included 240 HIV-negative men of the Amsterdam Cohort Study among MSM. We distinguished three casual partner types-one-night stand ("met by chance and had sex only once"); multiple-time casual partner ("met by chance and had sex several times"); and sex buddy ("contacted on a regular basis for sex but not considered a steady partner"). Serosorting was defined as UAI with an HIV-negative partner. GEE analyses were performed to examine the association between casual partner type and sexual risk behaviour.

Results 240 participants accounted for 362 reports of anal intercourse, of which 206 (57\%) with one-night stands, 95 (26\%) with multiple-time casual partners, and $61(17 \%)$ with sex buddies. The proportion of UAI was $19 \%$ (39/206) with one-night stands, $20 \%$ (19/95) with multiple-time casual partners, and 34\% (21/61) with sex buddies. When performing UAI, 21\% (8/39) reported serosorting with one-night stands, $42 \%(8 / 19)$ with multiple-time casual partners, and $52 \%(11 / 21)$ with sex buddies. Analyses revealed that only men with a sex buddy were significantly more likely to have UAI (OR [95\% CI] 2.39 [1.39 to 4.09]), but were also more likely to practice serosorting than men with a one-night stand (OR $[95 \% \mathrm{CI}]$ 5.20 [1.20 to 22.52]). No differences were found between the multiple-time casual partner and the other partner types.

Conclusions Men with a sex buddy had more UAI but also reported more serosorting than men with a one-night stand. As a result, the proportion of UAI without serosorting is lower for men with a sex buddy, and therefore men might have less UAI at risk for HIV with this partner type. However, the quality of serosorting with casual partners and hence their protective value against HIV needs to be further established. At this time, we suggest that a distinction between the one-night stand and the sex buddy should be incorporated in future studies as risk behaviour of MSM is significantly different between these partner types.

\section{P1-S2.48 EXPONENTIAL GROWTH OF LYMPHOGRANULOMA VENEREUM DIAGNOSES IN THE UK: INVESTIGATION OF THE LARGEST DOCUMENTED OUTBREAK AMONG MEN WHO HAVE SEX WITH MEN}

doi:10.1136/sextrans-2011-050108.105

G Hughes, S Alexander, I Simms, S Conti, C Powers, C Ison. Health Protection Agency, London, UK

Background With over 1500 cases reported, the UK now has the largest documented outbreak of Lymphogranuloma venereum (LGV) among men who have sex with men (MSM). Of particular concern is the recent exponential rise in cases - a third of all cases have been reported since 2010. We investigated the characteristics of this outbreak and specifically whether any factors were associated with the sharp upsurge in cases in 2010.

Methods The UK LGV Incident Group launched a diagnostic reference service and enhanced surveillance of LGV in the UK in October 2004. All symptomatic patients attending UK GUM clinics who were chlamydia-positive or contacts of positive cases were referred to STBRL in London and later also to the Scottish Bacterial Sexually Transmitted Infections Reference Laboratory in Edinburgh for confirmation. Enhanced LGV surveillance forms were completed by clinicians caring for cases and submitted to the HPA for analysis. An epidemic curve was plotted and the point of exponential growth estimated. The characteristics of cases prior to and during the exponential growth phase were compared.

Results Since 2003, 1570 LGV cases have been reported in the UK, of which 1268 (81\%) had enhanced surveillance forms available. All but 8 (99\%) were in MSM and most (77\%) were seen in London, Brighton and Manchester. The point of exponential growth was estimated as 1st October 2009. Compared to cases prior to this date, cases in the growth phase were more likely to be older (41\% vs $38 \%$ aged over 40 ), to be HIV positive ( $84 \%$ vs $78 \%$ ), to be HCV antibody positive ( $27 \%$ vs $22 \%$ ), to report unprotected anal intercourse (UAI) ( $80 \%$ vs $77 \%$ ) and fisting (19\% vs $14 \%$ ), to present outside London ( $32 \%$ vs $27 \%$ ), and to have acquired LGV in the UK (91\% vs $87 \%$ ). Overall, median case age was 38, $84 \%$ were white and $11 \%$ reported they acquired the infection outside the UK, mostly in Western European countries associated with the epidemic. UAI was reported by $78 \%$ of cases and at least 50 were identified as re-infections.

Conclusions LGV is becoming endemic in the UK and is characterised by HIV positive MSM involved in dense sexual networks. Although cases associated with the recent upsurge are more geographically widespread, there is no evidence of dispersal among lower risk or HIV negative MSM. LGV control will require intensified awareness raising and outreach among at risk populations. Regression modelling of key variables is underway to investigate and validate these findings.

\section{P1-S2.49 CHLAMYDIA AND GONORRHOEA TRANSMISSION AMONG MEN WHO HAVE SEX WITH MEN IN AMSTERDAM, IS INDEPENDENT OF NETWORK FACTORS OR PARTNERSHIP CHARACTERISTICS}

doi:10.1136/sextrans-2011-050108.106

${ }^{1} \mathrm{~A}$ Matser, ${ }^{2} \mathrm{~T}$ Heijman, ${ }^{1} \mathrm{R}$ Geskus, ${ }^{1} \mathrm{H}$ de Vries, ${ }^{1} \mathrm{H}$ Fennema, ${ }^{1} \mathrm{M} \mathrm{S}$ van der Loeff. ${ }^{1}$ Public Health Service, Amsterdam, Netherlands; ${ }^{2}$ Cluster of Infectious diseases, Department of research, Amsterdam, Netherlands

Background Partnerships are essential for transmission of sexually transmitted infections (STI). Among MSM, variation in partnerships is common. We have analysed risk factors for STI by studying partnerships.

Methods The study population was recruited from the STI outpatient clinic of the public health service of Amsterdam, the Netherlands. Inclusion criteria were male gender, having had sexual contact with men in the past 6 months, age $\geq 18$ years, and understanding of written Dutch or English. Recruitment occurred from July 2008 to August 2009. Participants were screened for chlamydia (CT), gonorrhoea (NG), syphilis, and HIV (opting-out strategy). Participants completed a questionnaire including demographics and detailed questions about sexual behaviour in self-defined relationships with a steady partner and the last three other partners within the previous 6 months. Logistic regression analysis was used to identify risk factors for CT and NG.

Results 2731 MSM reporting 7397 partnerships were included; median age was 39 years (IOR 31-45). CT prevalence was $12.5 \%$ $(n=342)$, NG prevalence was $11.1 \%(n=303)$, and CT/NG coinfection was found in $2.6 \%(n=71)$ of the MSM. Meeting partners online was common (53\% met at least one of the partners online), but not associated with STI (CT-OR 1.2 95\% CI 0.95 to 1.5 and NG-OR $1.295 \%$ CI 0.9 to 1.5), just as having concurrent partnerships (CT-OR 3.2 95\% CI 0.4 to 23.6 and NG-OR $1.395 \% \mathrm{CI}$ 0.3 to 5.6). In multivariable analysis, independent predictors of CT were having $=5$ known partners in the last 6 months (OR $1.795 \%$ CI 1.2 to 2.3), receptive unprotected anal intercourse (OR $2.195 \%$ CI 1.5 to 2.8 ), NG coinfection (OR $2.095 \%$ CI 1.5 to 2.8), and HIV coinfection (OR $1.895 \%$ CI 1.4 to 2.4). Independent predictors of NG were group sex (OR $1.595 \%$ CI 1.1 to 2.1), being in a different 10 -year age category than the partner (OR $1.495 \%$ CI 1.04 to 1.8), CT coinfection (OR $2.195 \%$ CI 1.5 to 2.8), and HIV coinfection (2.2 $95 \%$ CI 1.6 to 2.9 ). Age $=45$ years (OR $0.495 \%$ CI 0.2 to 0.6 ) and being bear type (OR $0.495 \%$ CI 0.2 to 0.9 ) were associated with lower NG risk. 